\title{
Gestão ambiental conceitual para o desenvolvimento sustentável do distrito industrial de Icoaraci (Belém/PA): estudo da vulnerabilidade das águas subterrâneas
}

Este estudo tem por objetivo central estudar a vulnerabilidade intrínseca das águas subterrâneas do local aplicando o método Groundwater occurance, Overall of lithology e Depth of groundwater (GOD) para a adaptação e transformação de um distrito industrial em operação em Parque Industrial Ecológico (PIE), no âmbito da gestão ambiental da Ecologia Industrial (EI). Apresenta-se uma breve revisão da literatura acerca dos distritos industriais, assim como os princípios da Ecologia Industrial. A partir da metodologia, a qual se baseia no estudo de profundidade de aquífero, sua condição de confinamento e a litologia da zona não saturada. Como fonte de dados foram usados os poços disponibilizados pela CPRM, por meio do SIAGAS e estudos científicos acerca de PIE e SI. Analisando-se a existência de indícios da cultura de El nos empreendimentos do distrito industrial. Os índices mostram que cerca de $70 \%$ da área apresenta vulnerabilidade baixa e $20 \%$ da área, é média. A principal justificativa para este contexto é a presença de argila empacotando os extratos arenosos. Conclui-se que a maior parte da área do Distrito Industrial de Icoaraci (Belém-PA) possui baixa vulnerabilidade, mas que ainda assim, as áreas de médias vulnerabilidades precisam de estudos mais detalhado para aumentar a segurança com as águas subterrâneas e os resultados obtidos indicaram que o distrito industrial de Icoaraci apresenta um potencial regular para ser adaptado em um PIE, conforme a analise proposta neste estudo.

Palavras-chave: Águas Subterrâneas; Vulnerabilidade; GOD; Distrito Industrial; Parque Industrial ecológico.

\section{Conceptual environmental management for the sustainable development of the industrial district of Icoaraci (Belém/PA): study of the vulnerability of groundwater}

The main objective of this study is to study the intrinsic vulnerability of the local groundwater using the Groundwater occurance, Overall of lithology and Depth of groundwater (GOD) method for the adaptation and transformation of an industrial district in operation in an Ecological Industrial Park (PIE), within the scope of the environmental management of Industrial Ecology (EI). A brief review of the literature on industrial districts is presented, as well as the principles of Industria Ecology. Based on the methodology, which is based on the study of aquifer depth, its confinement condition and the lithology of the unsaturated zone. As a source of data, the wells provided by CPRM were used, through SIAGAS and scientific studies about PIE and SI. Analyzing the existence of evidence of El culture in the industrial district enterprises. The indices show that about $70 \%$ of the area has low vulnerability and $20 \%$ of the area is average. The main justification for this context is the presence of clay packaging the sandy extracts. It is concluded that the majority of the area of the Industrial District of Icoaraci (Belém-PA) has low vulnerability, but that even so, the areas of medium vulnerability need more detailed studies to increase safety with groundwater and the results obtained indicated that the industrial district of Icoaraci has a regular potential to be adapted in a PIE, according to the analysis proposed in this study.

Keywords: Groundwater; Vulnerability; GOD; Industrial District; Ecological Industrial Park.

Topic: Planejamento, Gestão e Políticas Públicas Ambientais

Received: 12/03/2020 Approved: 09/04/2020

Reviewed anonymously in the process of blind peer.

Ana Carla Leite Carvalho Cabral (iD)

Universidade da Amazônia, Brasil

http://lattes.cnpq.br/6538382004505294

http://orcid.org/0000-0002-1735-1320

calincarvalho@gmail.com

Leonardo Augusto Lobato Bello

Universidade da Amazônia, Brasil http://lattes.cnpq.br/5449459117793556

http://orcid.org/0000-0002-2017-5292

leonardo.bello@unama.br

Maria do Socorro Bezerra Lopes

Universidade da Amazônia, Brasil

http://lattes.cnpq.br/1850698138869190

http://orcid.org/0000-0003-1650-4724

soclopes@gmail.com

DOI: 10.6008/CBPC2318-2881.2020.002.0009

\author{
David Franco Lopes (iD) \\ Universidade da Amazônia, Brasil \\ http://lattes.cnpq.br/4561329364320659 \\ http://orcid.org/0000-0002-5228-4418 \\ davidlopes.1978@gmail.com \\ Marco Aurélio Arbage Lobo \\ Universidade da Amazônia, Brasil \\ http://lattes.cnpq.br/6769430330686533 \\ http://orcid.org/0000-0002-3366-4992 \\ lobo2502@gmail.com
}

Referencing this:

CABRAL, A. C. L. C.; BELLO, L. A. L.; LOPES, M. S. B.; LOPES, D. F.; LOBO, M. A. A.. Gestão ambiental conceitual para o desenvolvimento sustentável do distrito industrial de Icoaraci (Belém/ PA): estudo da vulnerabilidade das águas subterrâneas. Nature and Conservation, v.13, n.2, p.82-93, 2020. DOI: http://doi.org/10.6008/CBPC23182881.2020.002.0009 


\section{INTRODUÇÃO}

As águas subterrâneas são importante fonte de águas potáveis, seja para consumo humano ou uso industrial, muitas vezes sendo a única fonte disponível numa região árida, sem corpos d'água superficiais. A ocorrência de um polo industrial, por congregar diversas atividades potencialmente poluidoras, sempre deve estar associado a perspectiva de boas práticas ambientais. O conceito de distrito industrial foi desenvolvido no final do século XIX por Alfred Marschall, no contexto da economia britânica da época (DAMAS, 2008). Historicamente, diversas modalidades de distritos industriais se conformaram em termos de localização, estrutura e tipos de produtos. De modo geral, um distrito industrial consiste em uma rede de indústrias concentradas em determinado local, onde se materializam as relações industriais, utilizando-se mão-de-obra e insumos, originando produtos e obtendo economias de escala locais. Mais recentemente, diversos espaços industriais caracterizados como distritos industriais passam por um processo de desagregação ou de reconfiguração produtiva, dando origem às redes de empresas, conectadas através de fluxos de bens, serviços e informação com o objetivo de fabricar um produto, e às cadeias produtivas, compostas pelas empresas produtoras e outros agentes como varejistas e organizações financeiras. No entanto, os distritos industriais ainda permanecem como arranjos produtivos predominantes especialmente nos países em desenvolvimento, como o Brasil. A lógica de implantação destes distritos industriais está historicamente pautada em fatores locacionais relacionados à disponibilidade de infraestrutura, mão de obra, dentre outros. Nesse contexto, os aspectos ambientais nem sempre estão presentes na definição da localização industrial. O conceito de Ecologia Industrial (EI) não é recente, pois começou a se desenvolver em meados da década de 1970 e se tornou mundialmente conhecido no início dos anos 1990, após a publicação do artigo 'Strategies for Manufacturing', de Robert Frosch e Nicholas Gallopoulos, na revista Scientific American, em 1989.

Porém, hoje a El ganha uma particular importância devido ao contexto do desenvolvimento sustentável e, também, por estar relacionada à Economia Circular, que busca substituir o modelo linear de produção de bens. A análise dos parques industriais brasileiros resulta na conclusão de que o modelo de desenvolvimento adotado ainda é o tradicional, representado por um sistema aberto, o qual depende de um suprimento contínuo e inesgotável de matéria e energia que, depois de utilizada, é devolvida ao meio ambiente. Soma-se a esse fato a necessidade de as indústrias nacionais elaborarem estudos prospectivos na área ambiental, referentes à reciclagem e reutilização de subprodutos em parques industriais. Para se atingir um modelo de desenvolvimento considerado 'sustentável', o mesmo deve ser representado por um sistema fechado, ou seja, um sistema onde os impactos oriundos das atividades produtivas retornam ou são recuperados, por meio de ações restauradoras que os minimizem. Assim, teoricamente um sistema fechado não geraria saídas nem devolveria resíduos ao meio ambiente. Alguns autores enfatizam que as organizações podem causar diversos tipos de impactos ambientais e ecológicos no meio ambiente. Entre as organizações industriais, comerciais e prestadoras de serviço, as organizações que mais impactam o meio ambiente são do ramo industrial, uma vez que possuem a característica de converter insumos produtivos em produtos finais. Diante do exposto, a maior justificativa da escolha deste tema é o conhecimento de que os Parques 
Industriais Ecológicos (PIEs) possuem os elementos e o potencial necessários para introduzir mudanças e melhorias na atual estrutura industrial de uma região, contribuindo por meio de uma gestão ambiental cooperativa para o desenvolvimento sustentável.

Dentre estas, planejar a prevenção de possíveis acidentes que possam colocar as águas subterrâneas em condições inadequadas ao consumo humano. Com esta compreensão esta pesquisa faz uma abordagem da vulnerabilidade natural das águas subterrâneas do Distrito Industrial de Icoaraci, na capital paraense, onde estão localizadas 31 empresas com grande riqueza de águas subterrâneas, cujos aquíferos Pirabas e Barreiras são os principais mananciais, sendo este último, objeto de análise neste trabalho, por estar mais próximo a superfície e ser mais explorado, conforme visualizado na Figura 1.

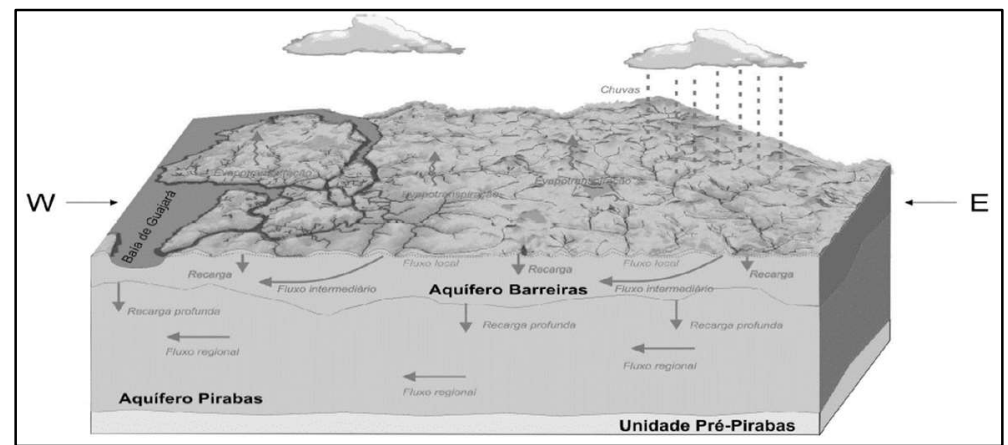

Figura 1: Aquífero Conceitual do sistema Barreiras - Pirabas. Fonte: ANA (2018).

A Formação Barreiras é caracterizada por depósitos de sedimentos siliciclásticos de cores variadas (SÁ, 1969). Aflora nas porções sudoeste, sudeste e norte da região metropolitana de Belém (RMB), incluindo na região de estudo, além de mostrar associada a crostas lateríticas ferruginosas.

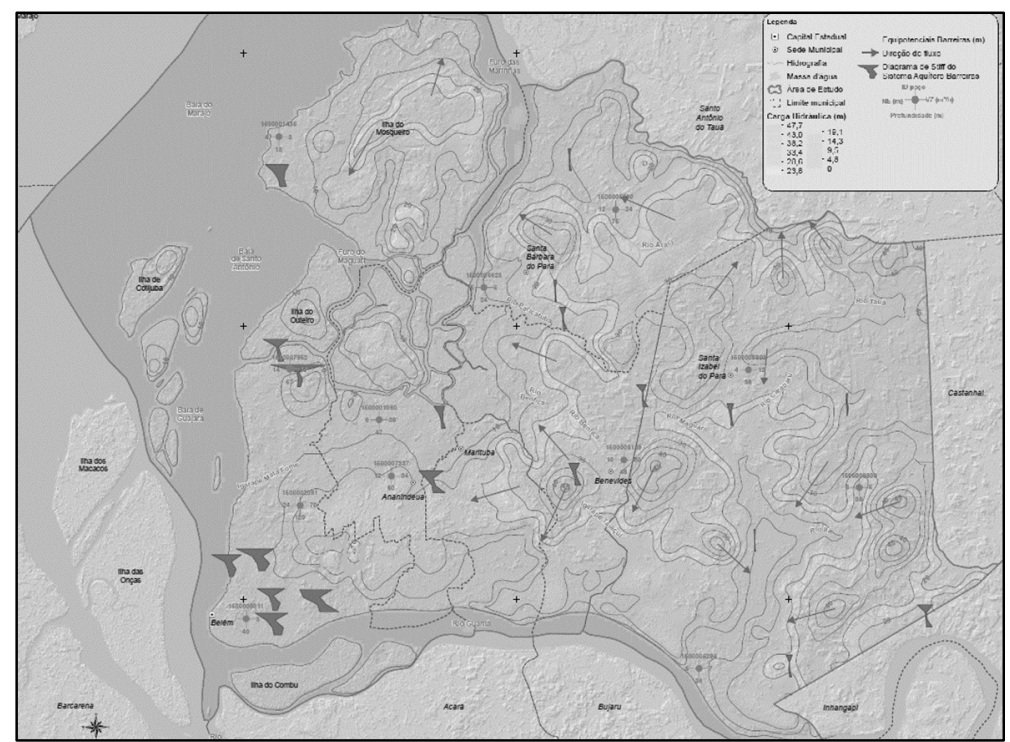

Figura 2: Mapa Hidrogeológico do Aquífero Barreiras. Fonte: modificado de ANA (2018).

Os sedimentos Pós-Barreiras correspondem aos depósitos mais recentes de latossolos amarelados e arenosos, finos a médios, em geral maciços, sobrepostos à Formação Barreiras (ROSSETI et al., 2013). Afloram nas porções nordeste, centro e noroeste da RMB e incluem depósitos pleistocênicos de paleocanais fluviais, eólicos e praias; e depósitos holocênicos representados por aluviões, colúvios e elúvios (ANA, 2018). Na 
Figura 2, se apresenta o mapa hidogeológico do Aquífero Barreiras, onde se identifica o poço 1500007952 , situado na área de estudo e que apresenta $57 \mathrm{~m}$ de profundidade, $14 \mathrm{~m}$ de nível estático e $30 \mathrm{~m}^{3} / \mathrm{h}$ de vazão.

Já a Formação Pirabas compreende uma sequência de rochas de composição carbonática com intercalações secundárias de folhelhos negros a esverdeados e arenitos amarelados. Essa unidade ocorre somente em subsuperfície na área estudada, protegida pela Formação Barreiras. No estado do Pará, essas rochas afloram na região litorânea do Nordeste do estado, nos municípios de Salinópolis, Capanema e São João de Pirabas, de onde derivou a sua denominação.

A Figura 3 mostra o mapa hidogeológico do Aquífero Pirabas, onde se identificam dois poços na área de estudo, o poço 1500001758 , com $300 \mathrm{~m}$ de profundidade, $11 \mathrm{~m}$ de nível estático e $285 \mathrm{~m}^{3} / \mathrm{h}$ de vazão; e outro de ID 1500007636 , com $100 \mathrm{~m}, 17 \mathrm{~m}$ e $73 \mathrm{~m}^{3} / \mathrm{h}$, respectivamente.

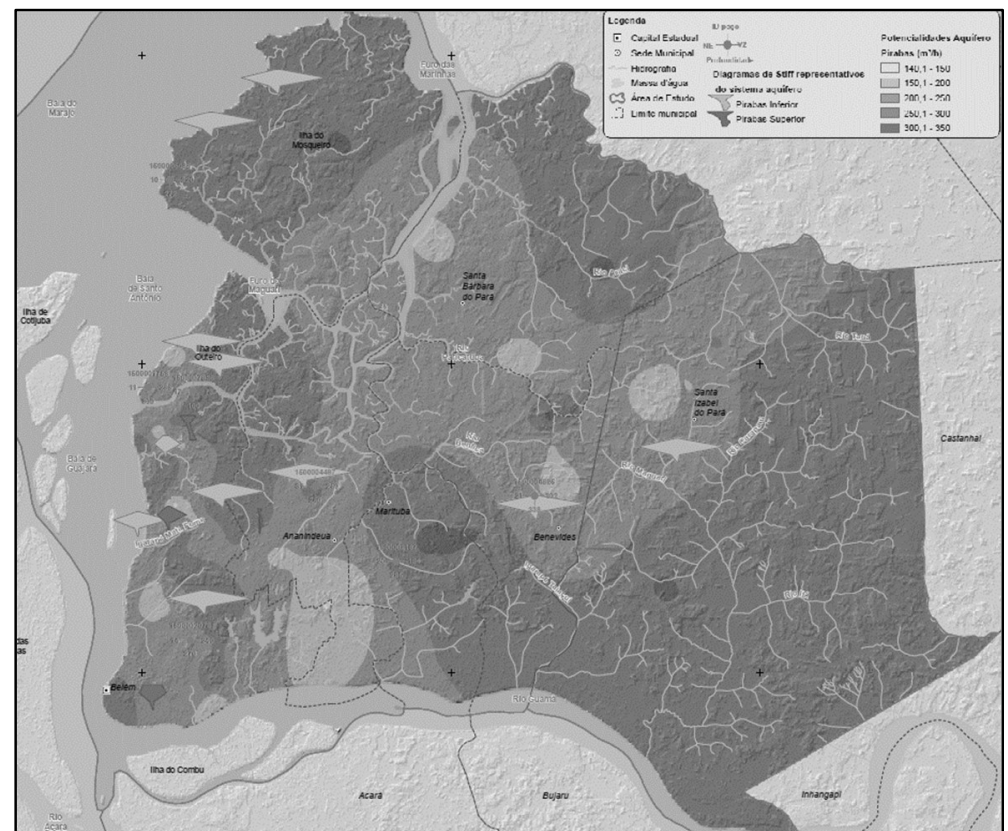

Figura 3: Mapa Hidrogeológico do Aquífero Pirabas. Fonte: modificado de ANA (2018).

Foi utilizada a base de dados pré-existentes do Sistema de Informações de Águas Subterrâneas Serviço Geológico do Brasil (SIAGAS - CPRM), disponível para acesso público em siagasweb.cprm.gov.br, analisando os dados com os devidos ajustes, conforme Santos et al. (2013).

. A base de dados foi adquirida por meio de downloads gratuitos, como o Sistema Estadual de Geoinformação do Estado de Goiás (SIEG) e pela NASA (Earth Explorer), onde foi possível a aquisição da imagem do Landsat 8. Anais XVII Simpósio Brasileiro de Sensoriamento Remoto - SBSR, João Pessoa-PB, Brasil, 25 a 29 de abril de 2015, INPE 7406 O método empregado para a obtenção do mapa de vulnerabilidade foi o GOD, onde foi amplamente testado graças a sua simplicidade conceitual e de aplicação (Foster e Hirata, 1998). O método GOD (FOSTER et al., 2002), o qual leva em consideração 3 parâmetros: o tipo de aquífero (G), a litologia e o grau de consolidação da Zona vadosa (O) e a profundidade do aquífero (D). O objetivo do método é a vulnerabilidade natural dos aquíferos, sem levar em consideração o tipo de contaminação que este pode estar sofrendo. A figura 2 demonstra o esquema utilizado para a aplicação do método. 


\section{MATERIAIS E MÉTODOS}

O método mais frequentemente usado para promover estratégias para prevenir a contaminação da água subterrânea, tem sido o Groundwater occurance, Overall of lithology e Depth of groundwater (GOD), para a realização das análises da vulnerabilidade de aquíferos à contaminação da área estudada, foram empregadas técnicas de geoprocessamento e sensoriamento remoto, aplicado ao estudo da vulnerabilidade do aquífero destaca-se o proposto por Foster et al. (1988) e rediscutido em Foster et al. (2002). Tal método indica o índice e grau de vulnerabilidade natural do aquífero por meio de três variáveis (profundidade do nível da água, litologia da zona não saturada e tipo e grau de confinamento aquífero).

Uma das principais ferramentas para proteger as águas subterrâneas, prevenindo sua contaminação, consiste em conhecer a vulnerabilidade natural do aquífero, a qual representa sua maior suscetibilidade a ser adversamente afetado por uma carga contaminante. Embora a relevância dessa atividade para o desenvolvimento econômico de determinada região, sua pratica intensa e de modo aleatório pode acarretar a contaminação das águas subterrâneas e a consequente proibição de seu uso em diversas atividades econômicas e ao consumo humano. Nesta perspectiva a pesquisa teve como objetivo mapear a vulnerabilidade natural do aquífero do Distrito Industrial de Icoaraci (Belém/PA). Para tanto utilizou-se na etapa inicial uma seleção de poços, inseridos no banco de dados do Sistema de Informações de Águas Subterrâneas (SIAGAS) e mantido a partir de mapeamento e de pesquisa hidrogeológica em todo país, é composto por uma base de dados de poços permanentemente atualizada e de módulos capazes de realizar consultas, pesquisas, extração e geração de relatórios, com perfis lito-construtivos disponíveis que contêm informações a respeito da espessura da zona não saturada e seus respectivos tipos litológicos e condição de confinamento.

De posse dos relatórios fornecidos pelo sistema da CPRM, foi possível obter os dados de grau de confinamento, litologia da zona não saturada e profundidade do nível freático. Relevante informar que os dados nem sempre são de fácil identificação e seu respetivo cálculo. Assim, foi necessário a interpolação de parâmetros e dimensões, tal qual proposto por Santos et al. (2013) e, somente após isto, estes dados foram ajustados e seus respetivos índices foram obtidos e em seguida o índice GOD foi calculado para o local de cada poço.

Todos estes dados foram consolidados em um novo banco de dados em planilha eletrônica plotados em mapa usando programa de geoprocessamento. Com o mapa, foram feitas visitas de campo, para entendimento contextualizado dos elementos naturais e construtivos da área, cujas informações serviriam de suporte à fase de interpretação dos dados retirados do SIAGAS e que serviram para análise integrada da vulnerabilidade intrínseca dos recursos hídricos subterrâneos.

Utilizou-se a seleção de poços, inseridos no banco de dados da CPRM que é gestor do SIAGAS e desenvolvido e mantido a partir de mapeamento e de pesquisa hidrogeológica em todo país, é composto por uma base de dados de poços permanentemente atualizada e de módulos capazes de realizar consultas, pesquisas, extração e geração de relatórios, com perfis lito-construtivos disponíveis que contêm informações 
a respeito da espessura da zona não saturada e seus respectivos tipos litológicos e condição de confinamento. Na área de estudo foram levantados 40 poços.

A metodologia G.O.D, concebida por Foster et al. (1988), permite a análise da susceptibilidade de um sistema aquíferos à contaminação através da vinculação entre três seguintes fatores: Groundwater hidraulic confinement: grau de confinamento hidráulico, ou seja, sua natureza ou condição de confinamento, se confinado, semi-confinado ou livre; Overlaying strata: tipos litológicos de estratos sobrejacentes ao meio saturado; Depth to groundwater table: profundidade do nível d'água, ou lençol freático para aquíferos livres, corresponde à espessura não saturada sobreposta ao aquífero.

Dessa maneira, a quantificação desse índice de vulnerabilidade compreende 3 etapas: 1. Identificação do grau de confinamento hidráulico do Aquífero Barreiras na área de estudo e as atribuições correspondente de peso que varia de 0,0 a 1,0; 2 . Definição dos caracteres litológicos da zona não saturada (peso entre 0,4 e 1 ), as quais foram obtidas, neste estudo, diretamente por dados de poços do SIAGAS. A atribuição de pesos de 0,4 a 1,0 é realizada mediante a ocorrência do tipo litológico em suas respectivas profundidades e avaliando sua predominância; 3 . Identificação da profundidade do nível freático e atribuições de pesos que variam de 0,6 a 1,0.

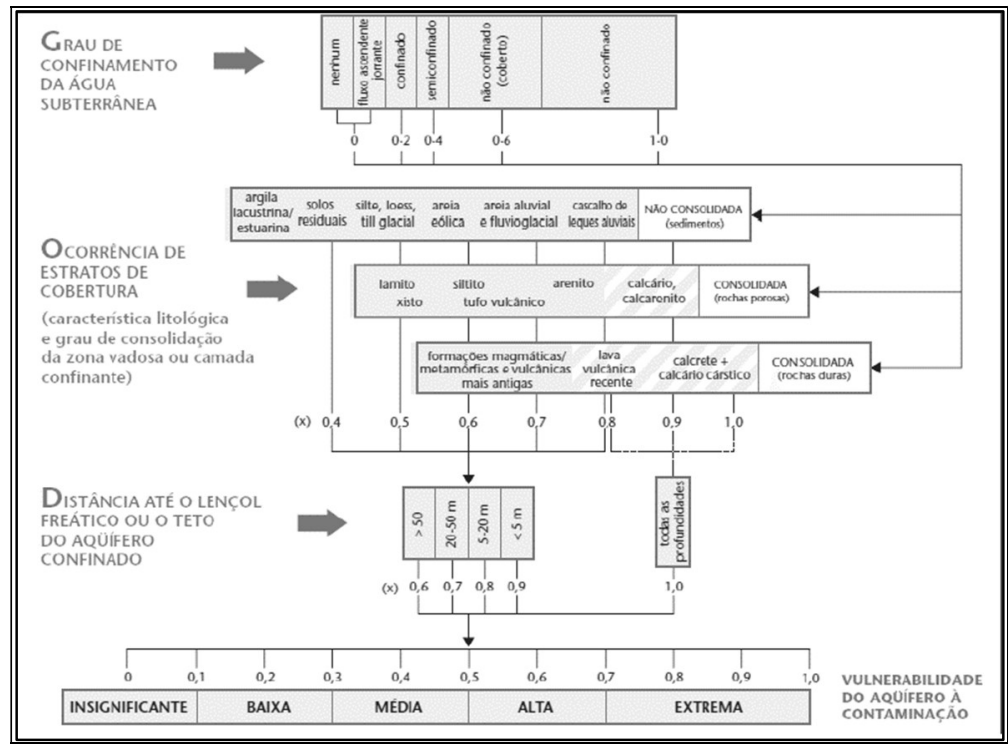

Figura 4: Diagrama explicativo da metodologia GOD. Fonte: Foster et al. (2002).

Após as definições dos pesos referentes a cada parâmetro, o índice de vulnerabilidade G.O.D é calculado pelo produto desses três valores $(G \times O \times D)$ em cada ponto examinado. Como resultado, tem-se magnitudes que variam de 0 a 1 , os quais assinalam as classes de vulnerabilidade natural, de modo crescente, na forma a saber desprezível, baixa, média, alta ou extrema (Figura4). 0 grau de confinamento (G) é fundamental para determinar a vulnerabilidade aquífera de uma região, pois é através da precipitação e posterior carreamento de sólidos pela água o contaminante pode possuir a capacidade de adentrar a zona não saturada.

Desta forma, a metodologia GOD foi aplicada, no presente estudo, de maneira a utilizar dados de natureza geológicas dos perfis de poços do SIAGAS. Os pesos atribuídos a cada fator seguiram a descrição 
litológica nos perfis de poços e a respectiva magnitude apresentada no método em si, avaliando-se a preponderância de cada litologia em cada ponto. Desse modo, foi atribuído peso de 0,9 a 0,2 ao parâmetro $\mathrm{G}$ em todos os pontos, caracterizando a natureza predominante do semi-confinado do aquífero na área. Os pesos atribuídos ao fator $O$ variam 0,18 a 1,4 de acordo com a descrição litológica e a predominância desses tipos em função de sua ocorrência nas espessuras não saturadas; e o peso variando de 0,2 a 0,9 foram imputados ao parâmetro $D$, de acordo com as espessuras não saturadas observadas nos dados.

A metodologia GOD apresenta resultados bastante satisfatórios em diversos contextos hidrogeológicos em termos de classificação de vulnerabilidade natural.

\section{RESULTADOS E DISCUSSÃO}

\section{Primeira etapa: Análise dos Parâmetros do SIAGAS}

De posse dos relatórios fornecidos pelo SIAGAS, foi possível obter os dados para implementar a metodologia GOD (Figura 4).

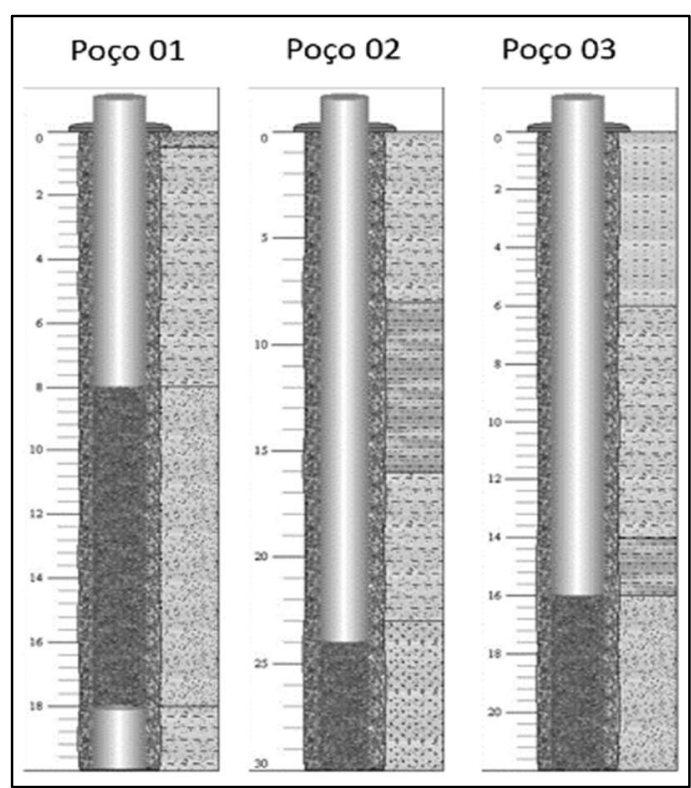

Figura 4: Perfis litoconstrutivos de poços usados como base do estudo de vulnerabilidade.

Para explicitar os resultados, os dados estão sendo apresentados na Tabela 1, a qual apresenta a sistematização de parte do banco de dados, onde está expresso o número do poço, o código do poço na base do SIAGAS, as coordenadas geográficas, o grau de confinamento, a litologia da zona não saturada, a profundidade do lençol freático, o índice GOD e sua significância (vulnerabilidade).

Tabela 1: Sistematização do banco de dados para obtenção da vulnerabilidade da água subterrânea.

\begin{tabular}{|l|l|l|l|l|l|l|l|}
\hline \multirow{2}{*}{ POÇO } & POÇO SIAGAS & \multicolumn{2}{l|}{ COORD GEOGR. } & TIPO AQUÍFERO & $\begin{array}{l}\text { LITOLOGIA } \\
\text { (O) }\end{array}$ & $\begin{array}{l}\text { PROF. } \\
\text { (D) }\end{array}$ & VULNERABILIDADE \\
\cline { 3 - 8 } & & LAT & LONG & (G) & 0,55 & 0,8 & MÉDIA \\
\hline PO01 & 1500005665 & 011814 & 482850 & 0,9 & 0,6 & 0,9 & ALTA \\
\hline PO02 & 1500001015 & 011805 & 482712 & 0,9 & 0,57 & 0,8 & INSIGNIFICANTE \\
\hline PO03 & 1500001025 & 011729 & 482742 & 0,9 & 0,39 & 0,8 & BAIXA \\
\hline PO04 & 1500001026 & 011712 & 482718 & 0,8 & 0,55 & 0,8 & MÉDIA \\
\hline PO05 & 1500001036 & 011657 & 482717 & 0,8 & 0,55 & 0,8 & MÉDIA \\
\hline PO06 & 1500001037 & 011717 & 482712 & 0,8 & & \\
\hline
\end{tabular}


Gestão ambiental conceitual para o desenvolvimento sustentável do distrito industrial de lcoaraci (Belém/ PA): estudo da vulnerabilidade das

CABRAL, A. C. L. C.; BELLO, L. A. L.; LOPES, M. S. B.; LOPES, D. F.; LOBO, M. A. A.

\begin{tabular}{|c|c|c|c|c|c|c|c|}
\hline $\mathrm{PO} 07$ & 1500001038 & 011717 & 482711 & 0,8 & 0,183 & 0,8 & BAIXA \\
\hline PO08 & 1500001039 & 011721 & 482711 & 0,8 & 0,31 & 0,8 & BAIXA \\
\hline PO09 & 1500001040 & 011718 & 482713 & 0,4 & 1,4 & 0,8 & MÉDIA \\
\hline P010 & 1500001041 & 011709 & 482715 & 0,8 & 0,55 & 0,8 & MÉDIA \\
\hline PO11 & 1500005664 & 011732 & 482741 & 0,4 & 0,45 & 0,4 & BAIXA \\
\hline $\mathrm{PO} 12$ & 1500005665 & 011729 & 482742 & 0,8 & 0,54 & 0,8 & ALTA \\
\hline $\mathrm{PO} 13$ & 1500005703 & 011654 & 482715 & 0,2 & 0,34 & 0,8 & INSIGNIFICANTE \\
\hline PO14 & 1500005714 & 011759 & 482655 & 0,2 & 0,2 & 0,8 & INSIGNIFICANTE \\
\hline PO- 15 & 1500005715 & 011800 & 482654 & 0,4 & 0,24 & 0,9 & INSIGNIFICANTE \\
\hline PO- 16 & 1500005716 & 011759 & 482654 & 0,4 & 0,4 & 0,9 & BAIXA \\
\hline PO- 17 & 1500005717 & 011755 & 482655 & 0,2 & 0,2 & 0,8 & INSIGNIFICANTE \\
\hline PO- 18 & 1500005719 & 011651 & 482726 & 0,9 & 0,68 & 0,9 & ALTA \\
\hline PO- 19 & 1500005765 & 011655 & 482636 & 0,4 & 0,502 & 0,8 & BAIXA \\
\hline PO- 20 & 1500006237 & 011722 & 482704 & 0,6 & 0,46 & 0,8 & BAIXA \\
\hline PO- 21 & 1500006431 & 011651 & 482718 & 0,6 & 0,46 & 0,8 & BAIXA \\
\hline PO- 22 & 1500006461 & 011726 & 482604 & 0,6 & 0,4 & 0,9 & BAIXA \\
\hline PO- 23 & 1500006538 & 011717 & 482710 & 0,6 & 0,49 & 0,8 & BAIXA \\
\hline PO- 24 & 1500006539 & 011731 & 482712 & 0,2 & 0,12 & 0,9 & INSIGNIFICANTE \\
\hline PO- 25 & 1500006540 & 011733 & 482711 & 0,6 & 0,52 & 0,8 & BAIXA \\
\hline PO- 26 & 1500006541 & 011735 & 482711 & 0,6 & 0,482 & 0,9 & MÉDIA \\
\hline PO- 27 & 1500006777 & 011729 & 482605 & 0,2 & 0,47 & 0,2 & INSIGNIFICANTE \\
\hline PO- 28 & 1500006793 & 011654 & 482655 & 0,2 & 0,56 & 0,2 & INSIGNIFICANTE \\
\hline PO- 29 & 1500007085 & 011723 & 482601 & 0,9 & 0,63 & 0,9 & ALTA \\
\hline PO- 30 & 1500007173 & 011729 & 482704 & 0,8 & 0,43 & 0,9 & MÉDIA \\
\hline PO- 31 & 1500007256 & 011755 & 482703 & 0,8 & 0,34 & 0,8 & MÉDIA \\
\hline PO- 32 & 1500007319 & 011730 & 482704 & 0,8 & 0,59 & 0,8 & MÉDIA \\
\hline PO- 33 & 1500007636 & 011652 & 482715 & 0,2 & 0,41 & 0,8 & INSIGNIFICANTE \\
\hline PO- 34 & 1500007798 & 011732 & 482711 & 0,8 & 0,77 & 0,9 & ALTA \\
\hline PO- 35 & 1500007799 & 011732 & 482710 & 0,2 & 0,48 & 0,8 & INSIGNIFICANTE \\
\hline PO- 36 & 1500007951 & 011739 & 482710 & 0,8 & 0,46 & 0,8 & MÉDIA \\
\hline PO- 37 & 1500007952 & 011738 & 482712 & 0,8 & 0,8 & 0,8 & INSIGNIFICANTE \\
\hline PO- 38 & 1500007953 & 011741 & 482711 & 0,9 & 0,9 & 0,9 & MÉDIA \\
\hline PO- 39 & 1500007957 & 011656 & 482653 & 0,9 & 0,45 & 0,9 & MÉDIA \\
\hline PO- 40 & 1500008460 & 011654 & 482631 & 0,9 & 0,45 & 0,9 & MÉDIA \\
\hline
\end{tabular}

\section{Segunda etapa: aplicação do método GOD}

Nesta etapa são mostrados os mapas que foram elaborados para cada parâmetro do método GOD: G - grau de confinamento; O - ocorrência do estrato de cobertura; e D - profundidade do aquífero. Estes permitiram uma análise de cada parâmetro do método, sendo possível a criação do mapa geral de vulnerabilidade.

O mapa do grau de confinamento apresenta classes ou faixas de valores (Figura 5). As áreas pretas do mapa apresentam maior dificuldade em infiltração de possíveis contaminantes/poluentes, por apresentarem localmente confinamento, cujas faixas variam de 0,2 a 0,43, que perfazem cerca de $5 \%$ da área $\left(0,39 \mathrm{~km}^{2}\right)$. As áreas acinzentadas, com valores de 0,44 até 0,61 , são aquelas que possuem possibilidades intermediárias de terem suas águas subterrâneas afetadas, serem semi-confinadas, e correspondem a cerca de $42 \%$ da área $\left(3,05 \mathrm{~km}^{2}\right)$. E as áreas brancas são as que apresentam maiores chances de infiltração, por serem não confinadas, cujos valores estão entre 0,62 a 0,9, e correspondem a cerca de $53 \%$ da área $(3,785$ $\left.\mathrm{km}^{2}\right)$. 


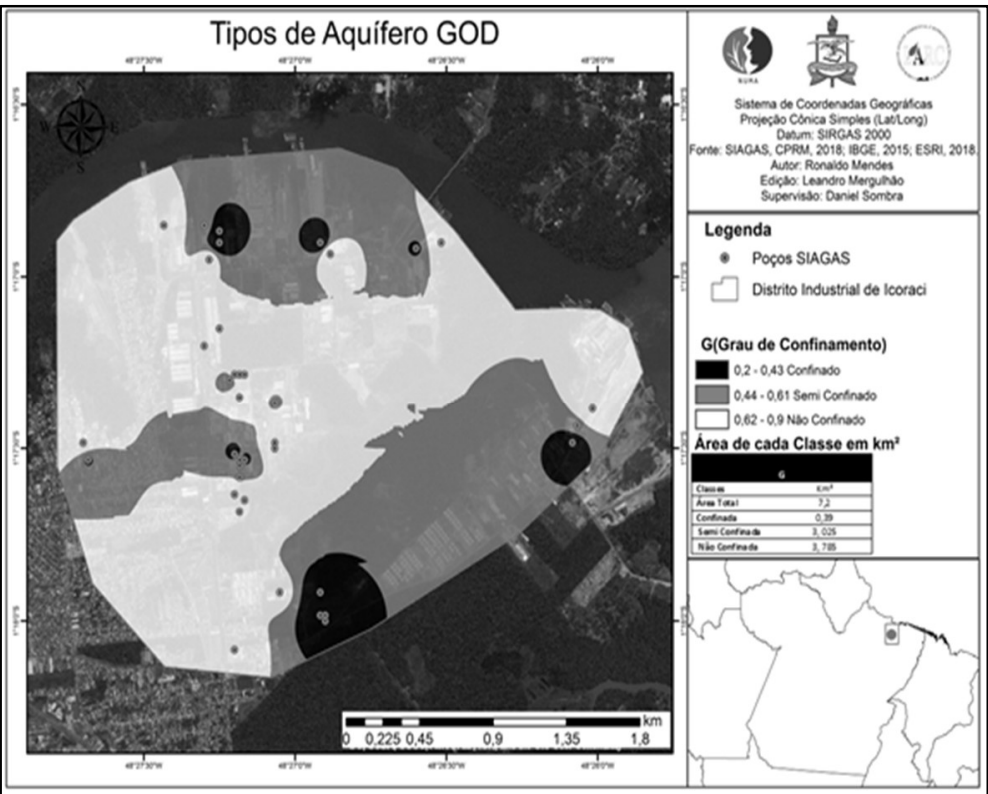

Figura 5: Mapa do Grau de confinamento do aquífero (G), conforme a parametrização do método GOD.

Tão importante quanto avaliar a vulnerabilidade é a ocorrência dos estratos de cobertura (O). Estes são responsáveis por conter ou facilitar o fluxo de possíveis fluidos prejudiciais a qualidade das águas subterrâneas. $\mathrm{Na}$ área em estudo são frequentes as variações entre estratos argilosos, arenosos, arenoargilosos e argilo-arenosos (Figura 6).

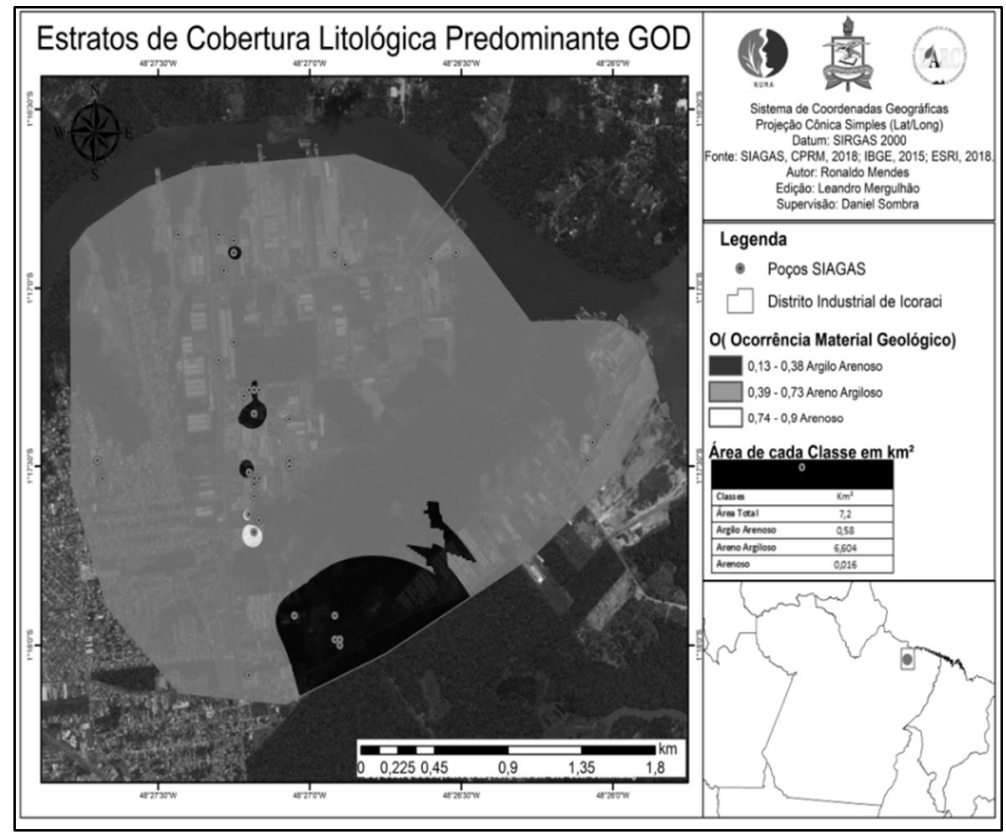

Figura 6: Mapa da Ocorrência dos estratos de cobertura (O), conforme a parametrização do método GOD.

As faixas de variação revelam a heterogeneidade litológica da área. O mapa apresenta faixas mais suscetíveis a infiltração, faixas menos suscetíveis e faixas intermediárias. As áreas pretas, são as menos suscetíveis a percolação de fluidos, por serem constituídas por material argilo arenoso, cujos valores variam de 0,13 a 0,39, e perfazem cerca de $8 \%$ da área $\left(0,59 \mathrm{~km}^{2}\right)$. As áreas cinzas têm suscetibilidade de percolação intermediária de fluidos, por serem constituídas por material areno argiloso, que apresentam faixas com de valores que variam de 0,39 a 0,73, e perfazem aproximadamente $92 \%$ da área $\left(6,604 \mathrm{~km}^{2}\right)$. Já a área branca 
possui a pior situação quanto a infiltração de prováveis poluentes, pois é constituída por material arenoso, cujos valores variam de 0,74 a 0,9, e perfazem apenas $0,2 \%$ da área $\left(0,016 \mathrm{~km}^{2}\right)$.

A profundidade do aquífero (D) é fator natural para o condicionamento da infiltração de qualquer fluido em subsuperfície. Assim, quanto maior a distância vertical entre a superfície e a água subterrânea, menos vulneráveis tais águas. Na área em questão (Figura 7), as profundidades variam de 2,84 m a 23,05 m. Desta forma abrangem três faixas: de 0 a $5 \mathrm{~m}$ de profundidade, com área branca no mapa, perfazem a maioria da área (cerca de $80 \%$ e valores de 0,81 a 0,9); de 5 a 20 m, com área cinza e com cerca de $20 \%$ da área e valores 0,61 a 0,8; e de 20 a $50 \mathrm{~m}$, com área preta cerca de $1 \%$ da área.

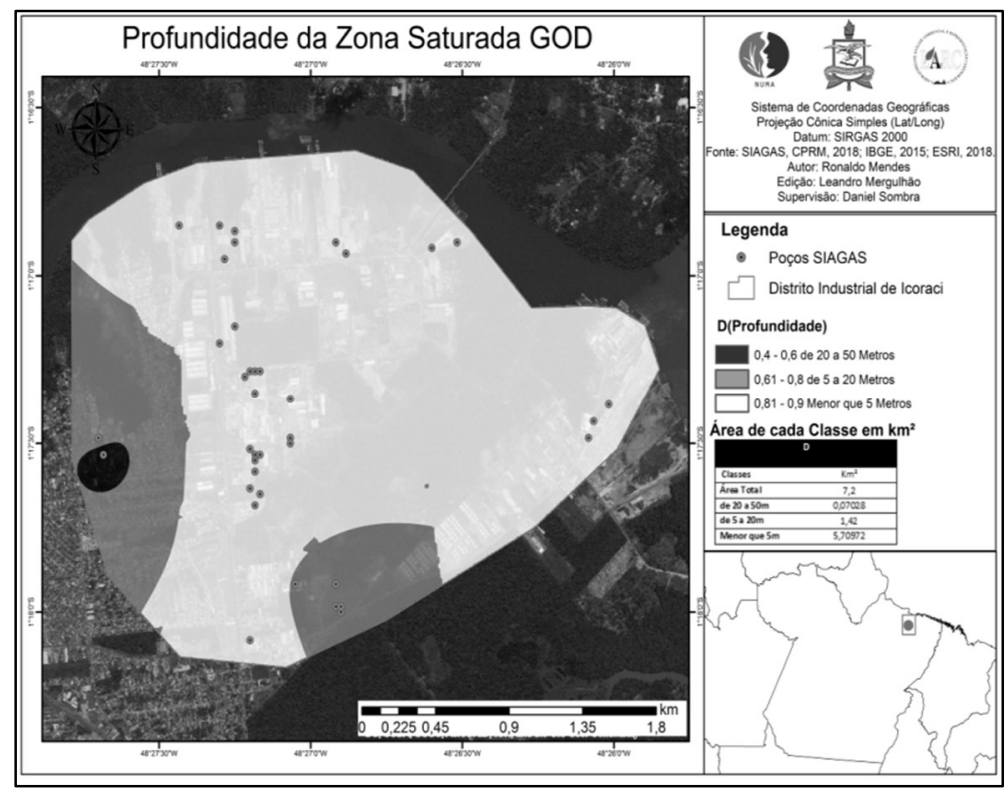

Figura 7: Mapa de profundidade da água subterrânea (D), conforme a parametrização do método GOD.

Diante dos mapas apresentados (Figuras 5, 6 e 7), é possível notar a variabilidade dos parâmetros levantados, o que naturalmente implica em heterogeneidade na vulnerabilidade, que se estende de insignificante, baixa, média e alta (Figura 8).

Quando observada a vulnerabilidade da água subterrânea expressa em mapa, é possível identificar facilmente a predominância em área da vulnerabilidade baixa (cinza escura), em cerca de $75 \%$ da área $(5,4$ $\mathrm{km}^{2}$, com valores entre 0,11 a 0,3). Em cerca de $23,1 \%$ da área $\left(1,66 \mathrm{~km}^{2}\right)$ está presente a vulnerabilidade média (cinza claro) (valores de 0,31 a 0,5), especialmente na porção Sul-Sudoeste e também ocorre em porções isoladas. Pontualmente também são identificadas vulnerabilidades insignificantes (preto, com cerca de $2 \%$ da área $-0,11 \mathrm{~km}^{2}$ e valores de 0,03 a 0,1 ) e alta (branco, com cerca de $0,4 \%$ da área $-0,03 \mathrm{~km}^{2} \mathrm{e}$ valores de 0,51 a 0,73$)$. 


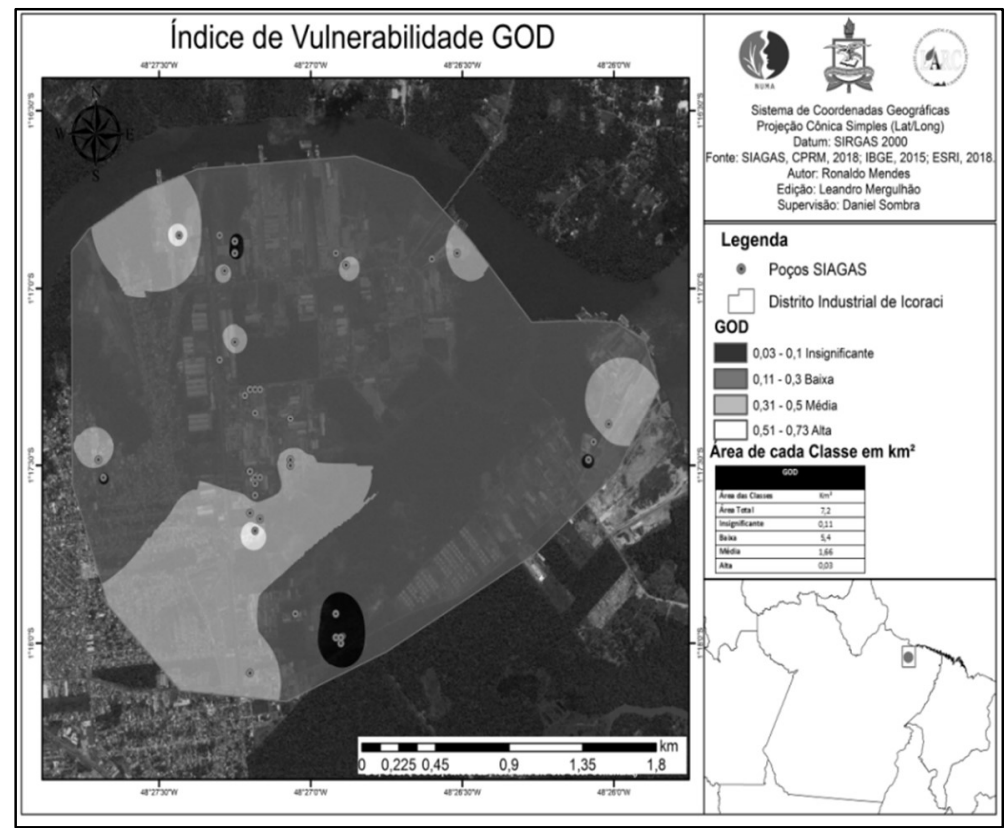

Figura 8: Mapa do índice de vulnerabilidade da água subterrânea obtida a partir do método GOD.

A predominância da vulnerabilidade baixa está vinculada especialmente a presença de argila nos perfis (especialmente areno argilosa), elementos frequentes na formação Barreiras da região (ROSETTI, 2006; ROSETTI et al., 1989), que mesmo que não configure confinamento, tem grande importância na proteção as águas subterrâneas.

\section{CONCLUSÕES}

Com base no trabalho realizado, concluiu-se que o estudo da vulnerabilidade intrínseca da água subterrânea do Distrito Industrial de Icoaraci (Belém/PA), mostra que cerca de $75 \%$ da área tem vulnerabilidade baixa e cerca de $23 \%$ apresenta vulnerabilidade média. Isto expressa que tal área apresenta condições favoráveis a destinação que Ihe foi dada. Naturalmente, melhor seria que as áreas fossem de vulnerabilidade insignificante. Ainda assim, é preciso estar atento as áreas cuja vulnerabilidade é média.

Diante do exposto o potencial necessário para introduzir mudanças e melhorias na atual estrutura industrial de uma região, contribuindo por meio de uma gestão ambiental cooperativa para o desenvolvimento sustentável. Sabendo que o uso do método GOD se presta a identificação da vulnerabilidade em uma dimensão com pouco detalhamento, sugere-se que, especialmente as áreas de vulnerabilidade média, seja objeto de detalhamento por meio de aplicação de métodos mais sofisticados.

\section{REFERÊNCIAS}

ANA. Agência Nacional de Água. Estudos Hidrogeológicos para a Gestão das Águas Subterrâneas da Região de Belém/PA da Agência Nacional de Águas. Brasília: ANA, 2018.

DAMAS, E. T.. Distritos industriais da cidade do Rio de Janeiro: gênese e desenvolvimento no bojo do espaço industrial carioca. Dissertação (Mestrado em Geografia) Universidade Federal Fluminense, Niterói, 2008.
FOSTER, S.; HIRATA, R.. Groundwater risk assessment, a methodology using available data. Pan American Heakth Organization, 1988.

FOSTER, S.; HIRATA, R.; GOMES, D.; D'ELIA, M.; PARIS, M.. Groundwater quality protection: a guide for water service companies, municipal authorities and environment agencies. Washington: World Bank, 2002. 
ROSETTI, D. F.. Evolução sedimentar miocênica nos estados do Pará e Maranhão. 2 ed. Inpe ePrint., 2006.

ROSSETTI, D. F.; BEZERRA, F. H. R.; DOMINGUEZ, J. M. L. Late Oligocene-Miocene transgressions along the equatorial and eastern margins of Brazil. Earth-Science Reviews, v.123, p.87-112, 2013.

ROSSETTI, D. F.; TRUCKENBRODT, W.; GÓES, A. M.. Estudo paleoambiental e estratigráfico dos sedimentos Barreiras e Pós-Barreiras na região bragantina, nordeste do Pará.
Boletim do Museu Paraense Emílio Goeldi, v.1, n.1, p.25-74, 1989.

SÁ, J. H. S.. Contribuição à Geologia dos sedimentos terciários e quaternários da região bragantina, Estado do Pará. Boletim Geológico (UFRJ), v.3, p.21-36, 1969.

SANTOS, A. C. B.; MENDES, R. L. R.; SILVA, G. N.; TAVARES, A. N.. Vulnerabilidade de aquíferos: uma análise da aplicação do método GOD com a base de dados Siagas. In: CONGRESSO INTERNACIONAL DE MEIO AMBIENTE SUBTERRÂNEO, 3. Anais. Revista Águas Subterrâneas, 2013.

A CBPC - Companhia Brasileira de Produção Científica (CNPJ: 11.221.422/0001-03) detém os direitos materiais desta publicação. Os direitos referem-se à publicação do trabalho em qualquer parte do mundo, incluindo os direitos às renovações, expansões e disseminações da contribuição, bem como outros direitos subsidiários. Todos os trabalhos publicados eletronicamente poderão posteriormente ser publicados em coletâneas impressas sob coordenação da Sustenere Publishing, da Companhia Brasileira de Produção Científica e seus parceiros autorizados. Os (as) autores (as) preservam os direitos autorais, mas não têm permissão para a publicação da contribuição em outro meio, impresso ou digital, em português ou em tradução. 